\title{
XXIX. On the range of Stokes's deep-water waves
}

\section{R.F. Gwyther}

To cite this article: R.F. Gwyther (1906) XXIX. On the range of Stokes's deep-water waves , Philosophical Magazine Series 6, 11:63, 374-378, DOI: 10.1080/14786440609463452

To link to this article: http://dx.doi.org/10.1080/14786440609463452

册 Published online: 16 Apr 2009.

Submit your article to this journal 준

Цlll Article views: 2

Q View related articles $₫$ 
Hence the term to be added to - EI $y$ is

$$
\frac{4}{\ddot{b}} \frac{I^{\prime}}{l}\left(\frac{x^{2}}{2}-l x+\frac{l^{2}}{2}\right)
$$

and for a first approximation,

$$
-\frac{\ddot{y}_{1}}{y_{1}}=\frac{\mathrm{EI}}{.0819 \dot{4} \rho \omega l^{4}+\cdot \dot{3} m l^{3}+\cdot \dot{6} \mathrm{I}^{\prime} l} \text {. }
$$

This example is sufficient to indicate the procedure to be adopted in all such cases.

University College, Bristol, October 1905.

XXIX. On the Range of Stokes's Deep-Water Waves. By R. F. GwYTHER *.

THE waves with which I propose to deal are the waves of 1 finite amplitude of which the investigation was initiated by Sir George Stokes $\dagger$ in his paper on the Theory of Oscillatory Waves, and continued in the Supplement to that paper. The object of the paper is to establish the correctness of the opinion expressed in the paper quoted (p. 227) "After careful consideration I feel satisfied ... that we may approach as near as we please to the form in which the curvature at the vertex becomes infinite, and the vertex becomes a multiple point where the two branches ... enclose an angle of $120^{\circ}$." The method of the Supplement is adhered to as closely as is convenient. The result is to establish that the velocity in all waves of the series, small as well as great, is represented by a function which possesses poles which as the amplitude increases approach nearer to the fluid surface, and in the limiting form is identical with that investigated by Mr. Michell $\ddagger$ in his paper on the "Highest Wave in Water," in which the poles lie on the water-surface.

$\$ 1$. Making a change in the notation used by Stokes in the Supplement, in order to make the analytical form of the velocity more obvious, I write

$$
x+i y=\frac{\phi+i \psi}{c}-i \Sigma \frac{h_{n}}{n k} e^{\frac{i n k(\phi+i \psi)}{c}},
$$

where $n$ is an integer, and $k$ is a constant defining the wavelength. Also let $\psi=0$ define the free surface of the water.

- Communicated by the Author.

+ Mathematical and Physical Papers, vol. i. p. 197, and Supplement, p. 314.

$\ddagger$ Phil. Mag. November 1893. 
Then the velocity is given by

where

$$
q e^{i \omega}=u+i v=\frac{1}{\frac{d(x-i y)}{d(\phi-i \psi r)}}=\frac{c}{f\left(\frac{\phi-i \psi}{c}\right)}
$$

$$
f\left(\frac{\phi+i \psi}{c}\right)=1+\Sigma h_{n} e^{\frac{i n k(\phi+i \downarrow)}{c}} .
$$

At the free surface we have $\psi=0$, and it will be convenient in this case to write, in place of $f\left(\frac{\phi+i \psi}{c}\right)$,

$$
\mathrm{F}\left(\frac{i k \phi}{c}\right)=1+\Sigma h_{n} e^{\frac{i n k \phi}{c}} \text {. . . . . . }
$$

The condition for uniform pressure along the free surface requires that, when $\psi=0$,

$$
g y-\frac{1}{2} q^{2}=\text { constant. }
$$

The determination of the successive values of $h_{n}$ resulting from this condition is only a matter of labour. I have carried the determination to the sixth order, as an extended number of terms is important as the basis of the future argument.

Stating results only, I find

$$
\begin{aligned}
k c^{2} / g & =1+h^{2}+\frac{7}{2} h^{4}+\frac{229}{12} h^{6}, \\
h_{2} & =2 h^{2}+h^{4}+\frac{29}{6} h^{6}, \\
h_{3} & =\frac{9}{2} h^{3}+\frac{19}{4} h^{5}, \\
h_{4} & =\frac{32}{3} h^{4}+\frac{313}{18} h^{6}, \\
h_{5} & =\frac{625}{24} h^{5}, \\
h_{6} & =\frac{324}{5} h^{6}, . . . . . . .
\end{aligned}
$$

where I have written $h$ in place of $h_{1}$.

Also if we write $2 \alpha$ to denote the height of the wave from trough to crest, I find

$$
k a=h+\frac{3}{2} h^{3}+\frac{163}{24} h^{5} . \text {. . . . . . }
$$


If we write out in full the resulting expression for $\mathrm{F}\left(\frac{i k \phi}{c}\right)$, the successive terms will be seen to show a marked resemblance to those in the expansion of

$$
\left(1-3 h e^{\frac{i k \phi}{c}}\right)^{-\frac{1}{3}}
$$

In order to examine this resemblance more closely, I expand $\left\{\mathrm{F}\left(\frac{i k \phi}{c}\right)\right\}^{-3}$ on the assumption of convergence, and find

$$
\begin{aligned}
& \left\{\mathrm{F}\left(\frac{i k \phi}{c}\right)\right\}^{-3}=1-3 h e^{\frac{i k \phi}{c}}-\left(3 h^{4}+\frac{29}{2} h^{6}\right) e^{2 i k \phi} \\
& +\left(\frac{1}{2} h^{3}-\frac{9}{4} h^{5}\right) e^{\frac{3 i k \phi}{c}}+\left(h^{4}-\frac{7}{6} h^{6}\right) e^{\frac{4 i k \phi}{c}}+\frac{15}{8} h^{5} e^{\frac{5 i k \phi}{c}} \\
& +\frac{18}{5} h^{6} e^{\frac{6 i k \phi}{c}} \text {. }
\end{aligned}
$$

It is on the greatly increased convergency produced in the series by this step that the argument depends. It is clear that there is a value of $\mu$, not very widely different from $3 h$, for which $1-\mu e^{\frac{i k \phi}{c}}$ is a factor of the right-hand side of (4).

For more precision, write

$$
3 h=\alpha \mu+\beta \mu^{3}+\gamma \mu^{5}
$$

and determine $\alpha, \beta, \gamma$ so that the right-hand side of (4) may vanish approximately when $1 / \mu$ is put in place of $e^{\frac{i k \phi}{e}}$, i.e. so as to satisfy

$$
1-\frac{3 h}{\mu}-\frac{3 h^{4}+\frac{29}{2} h^{6}}{\mu^{2}}+\ldots .=0 .
$$

This leads to the equations

$$
\begin{gathered}
1-\alpha+\frac{1}{54} \alpha^{3}+\frac{1}{81} \alpha^{4}+\frac{5}{648} \alpha^{5}+\frac{2}{402} \alpha^{6}=0, \\
\beta\left(1-\frac{1}{18} \alpha^{2}-\frac{4}{81} \alpha^{3}\right)=-\left(\frac{1}{27} \alpha^{4}+\frac{1}{108} \alpha^{5}+\frac{7}{4574} \alpha^{6}\right), \\
\gamma=-\left(\frac{4}{27} \alpha^{3} \beta+\frac{29}{1458} \alpha^{6}\right) .
\end{gathered}
$$


These give approximately

$$
\begin{aligned}
& \alpha=1.054, \\
& \beta=-.068, \\
& \gamma=-.015
\end{aligned}
$$

and these values lead to.

$$
h=\cdot 351 \mu-\cdot 023 \mu^{3}-\cdot 005 \mu^{5} . \quad . \quad . \quad .
$$

This value of $h$ increases with increasing fractional values of $\mu$, and reaches the value $\cdot 323$ approximately when $\mu=1$.

Since the hypothesis of convergence holds good for any value of $\mu$ finitely less than unity, we may take this to give approximately the greatest admissible value of $h$.

Using the numerical valnes (5) again, we find when $\mu<1$,

$$
\left\{\mathrm{F}\left(\frac{i k \phi}{c}\right)\right\}^{-1}=\left(1-\mu e^{\frac{i k \phi}{c}}\right)^{\frac{1}{3}} \times \mathrm{U}
$$

where

$$
\begin{gathered}
\mathrm{U}=1-\left(\cdot 018 \mu-.023 \mu^{3}-\cdot 005 \mu^{5}\right) e^{\frac{i k \phi}{c}}-\left(\cdot 019 \mu^{2}-\cdot 009 \mu^{c}\right) e^{\frac{2 i k \phi}{c}} \\
-\left(\cdot 011 \mu^{3}-.003 \mu^{5}\right) e^{\frac{3 i k \phi}{c}}-.006 \mu^{4} e^{\frac{4 i k \phi}{c}}-.003 \mu^{5} e^{\frac{5 i k \phi}{c} \ldots}
\end{gathered}
$$

The expression for the velocity $\left(q e^{i \omega}\right)$ will possess a series of poles, generally above the water, indicated by

$$
\mu e^{\frac{i k(\phi+i \psi)}{c}}=1 \text {. }
$$

Using the numbers in (2) and (3), I find

and

$$
k c^{2} / g=1+\cdot 123 \mu^{2}+\cdot 038 \mu^{4}+\cdot 019 \mu^{6}
$$

$$
k \alpha=\cdot 351 \mu+\cdot 041 \mu^{3}+\cdot 019 \mu^{5} .
$$

If we write $k=2 \pi / \lambda$, we get

$$
\frac{2 \alpha}{\lambda}=\cdot 112 \mu+\cdot 013 \mu^{3}+\cdot 006 \mu^{5}, \quad \cdot .
$$

which gives for the height of the greatest rounded wave $2 \alpha / \lambda=131$ approximately. This value is not greatly in defect of the ratio, viz. 142 , found by Mr. Michell for the pointed wave. There appears therefore to be no stage, while $\mu$ is finitely less than unity, at which the expressions cease to give rounded waves of the same general character.

$\$ 2$. In order to demonstrate that in the critical stage when Phil. Mag. S. 6. Vol. 11. No. 63. March 1906. 2 C 
$\mu=1$, this method leads to expressions identical with those of $\mathrm{Mr}$. Michell, I return to the general free-surface condition, and, for convenience, write $\zeta$ in place of $\phi / c$.

On differentiating the free-surface condition in respect to $\zeta$, we get an equation of the form

$$
\frac{u}{i}\{\mathrm{~F}(i k \zeta)-\mathrm{F}(-i k \zeta)\}=c^{2} \frac{d}{d \zeta}\{\mathrm{F}(i k \zeta) \mathrm{F}(-i k \zeta)\}-1 ;
$$

and in this I write

$$
\begin{aligned}
\mathrm{F}(i k \zeta) & =\left(1-\mu e^{i k \zeta}\right)^{-\frac{1}{3}} \mathrm{U}-1, \\
\mathrm{~F}(-i k \zeta) & =\left(1-\mu e^{-i k \zeta}\right)^{-1} \mathrm{~V}-1,
\end{aligned}
$$

where $\mathrm{U}$ and $\mathrm{V}$ are rapidly converging series of the type

so that

$$
1+\mathrm{C}_{1} e^{i i k \zeta}+\mathrm{C}_{2} e^{2 i k \zeta}+\ldots
$$

$$
\begin{aligned}
& \frac{g}{i}\left\{\frac{1}{\left(1-\mu \epsilon^{i i_{i}^{\sigma}}\right)^{\frac{1}{3}} \mathrm{U}}-\frac{1}{\left(1-\mu e^{-i K^{2}}\right)^{\frac{1}{3} \mathrm{~V}}}\right\} \\
& =c^{2} \frac{d}{d \xi}\left\{\left(1-\mu e^{i k \xi}\right)^{\frac{1}{3}}\left(1-\mu e^{\prime}-i k\right)^{\frac{1}{3}} U V\right\} . . . .
\end{aligned}
$$

This may be put in the form

$$
\begin{aligned}
& -\frac{g}{i}\left\{\left(\frac{1-\mu e^{i k \zeta}}{1-\mu e^{-i k \zeta}}\right)^{l} \mathrm{U}-\left(\frac{1-\mu e-i k \zeta}{1-\mu e^{i k \zeta}}\right)^{?} V\right\} \\
& =c^{2}\left\{\frac{\left(1-\mu e^{i \pi \zeta \zeta}\right)^{\frac{1}{2}}\left(1-\mu e^{-i k \zeta))^{\frac{1}{2}} d}\right.}{d \zeta}\left(\mathrm{U}^{2} V^{2}\right)\right. \\
& \left.+\frac{2 k \mu}{3} \frac{\sin k \zeta}{\left(1-\mu e^{i k \zeta}\right)^{\frac{1}{3}}\left(1-\mu \mu^{-i k \zeta)^{\frac{1}{3}}}\right.} \mathrm{U}^{2} V^{2}\right\} \text {. }
\end{aligned}
$$

which becomes, when $\mu=1$, identical, except for the change in units, with the equation which Mr. Michell has employed to obtain the form of the Highest Wave in Water.

If, however, the left-hand side of (9) is rationalized the right-hand side is also found to be rational, and this form may be found more convenient whether $\mu$ is less than or equal to unity. The values of the constants in $U$ and $V$ are, of course, different in these two cases.

The simplification on which I bave relied would appear to be applicable to all cases of irrotational waves whether in shallow or in deep water, and it will probably be found that such waves in either case will be related to poles, as I bave shown to be the case with deep-water waves. 